\title{
A Collaborative methodology intended for CBIR by means of contour
}

\author{
Tamilkodi.R ${ }^{\# 1}$, Roseline Nesa Kumari.G ${ }^{* 2}$, Maruthuperumal.S $S^{\# 3}$ \\ ${ }^{\# 1}$ Research Scholar, Saveetha University,Assoc.Prof,GIET, ${ }^{\# 3}$ Professor, NBKR Inst. of Science \& Tech. \\ Chennai, T.N, Rahajmundry,A.P, India, Nellore, A.P, India \\ tamil_kodiin@yahoo.co.in \\ 3 maruthumail@gmail.com \\ ${ }^{* 2}$ Professor, CSE, Saveetha School of Engineering, Chennai, T.N \\ 2rosemaruthu@gmail.com
}

\begin{abstract}
- content based image retrieval uses illustration stuffing to explore images from the huge range image database. Conventional methods of indexing and recovery using simply keywords have turned out to be obsolete. To trounce the problems faced in traditional methods we have developed substitute methods to portray images using their illustration content. For probing an image from the record, images need to be described by definite features. The most imperative attribute to depict an image is its shape. Narrative of Shape is denoted by assorted techniques which are in general separated into two broad categories boundary-based and region-based methods. in view of the fact that the human visual system itself focuses on edges and ignores identical regions, concentrates on boundary-based representations. Region based image retrieval techniques segment an image into a number of regions that work as local descriptor. Our proposed method RBBM (Region Boundary Based Method) employs these two different techniques collectively to give the accurate retrieval from large database with rapidity. The experimental results show the accuracy of retrieval when compared to other approaches.
\end{abstract}

Keyword- Content based image retrieval, boundary-based, region-based methods, shape.

\section{INTRODUCTION}

The weight of content-based retrieval for many applications, ranging from art galleries and museum archives to picture collections, criminal investigation, medical and geographic databases, makes the visual information retrieval one of the fastest budding research fields in information technology. Therefore, many content-based retrieval applications have been created for both research and commercial purposes.Early techniques of image retrieval were based on the manual textual annotation of images, a cumbersome and also often a subjective task. Texts alone are not sufficient because of the fact that interpretation of what we see is hard to characterize by them. Hence, contents in an image, color, shape, and texture, started gaining prominence. Initially, image retrievals used the content from an image individually. For example, Huang and Jean [5] used a 2D C+-strings and Huang et al. [3] used the color information for indexing and its applications. Approaches using a combination of contents then started gaining prominence. Combining shape and color using various strategies such as weighting [1], histogram-based [2], kernel-based [7], or invariance-based [8] has been one of the premier combination strategies.

In particular, image regions occupied by an object have to be found in order to describe its shape, and a number of known segmentation techniques combine the detection of low-level color and texture features with regiongrowing or split-and-merge processes. Choosing appropriate features for a shape recognition system must consider what kinds of features are suitable for the task. There exists no general feature which would work best for every kind of images. Shape features have very important role to play. Even if images with no color and no texture are to be retrieved based on features, in such case shape feature are required.

Shape is one of the most important visual attributes in an image. In fact, the human visual system is able to extract and abstract shapes from very complex scenes. The concept of shape is invariant to translations, rotations, and scaling, the shape of an object is a binary image representing the extent of the object. Due to these considerations shape presentation is one of the most challenging aspects of computer vision. Shape representations can be roughly classified in two major categories: boundary-based and region-based. The former represents shape by its outline, while the latter considers shape being formed of a set of two-dimensional regions. The main idea is to search for the regions in the image [15] looking for typical groups of statistically equally bright elements. First image is segmented based on a specific brightness represents a class of the image. Then, the features are extracted from the segmented classes [9]. Our proposed RBBM employ these two different techniques collectively to give the accurate retrieval from large database with rapidity. 
Our paper is organized as follows. Section 2 reviews the idea behind content based image retrieval using shapes which plays an important role in retrieval. Section 3 describes proposed (RBBM) techniques for Boundary based and region based. Experimental results with exactness tables are explained in section 4. Lastly conclusion is made in section 5 followed by the references.

\section{CONTOUR ASPECT}

Shape is an important visual feature and it is one of the basic features used to describe image content. However, shape representation and description is a difficult task. This is because when a 3-D real world object is projected onto a 2-D image plane, one dimension of object information is lost. As a result, the shape extracted from the image only partially represents the projected object. Basically, shape-based image retrieval consists of measuring the similarity between shapes represented by their features. Some simple geometric features can be used to describe shapes. Usually, the simple geometric features can only discriminate shapes with large differences; therefore, they are usually used as filters to eliminate false hits or combined with other shape descriptors to discriminate shapes. They are not suitable to stand alone shape descriptors. A shape can be described by different aspects [12].

The exploit of object shape is one of the mainly tough tribulations in creating proficient CBIR. The object's shape plays a vital role in incisive for related image objects. In image retrieval, one expects that the shape depiction is invariant to scaling, rotation, and translation of the object and is naturally either 2D or 3D depending on the object. Shape features are less developed than their color and texture counterparts for the reason that of the inherent intricacy of representing shapes. At present CBIR exploits two large groups of 2D shape descriptors, namely, boundary-based and region-based, representing either an outer boundary or an entire region. These representations can also be combined together to obtain more efficient retrieval.

\section{DISCRETE WAVELET TRANSFORM}

The 2-D discrete wavelet transforms (DWT) decomposes the image into sub-images, 3 details and 1 approximation. The approximation looks just like the original; only on 1/4 the scale. The 2-D DWT is an application of the 1-D DWT in both the horizontal and the vertical directions. The DWT separates an image into a lower resolution approximation image (LL) as well as horizontal (HL), vertical (LH) and diagonal (HH) detail components. The low-pass and high-pass filters of the wavelet transform naturally break a signal into similar (low pass) and discontinuous/rapidly-changing (high-pass) sub-signals. The slow changing aspects of a signal are preserved in the channel with the low-pass filter and the quickly changing parts are kept in the high-pass filter's channel.

A query image is fed into a wavelet filter bank and is decomposed into de-correlated sub bands. Each sub band captures the feature of some scale and orientation of the original image. The proposed TSW decompose an image into single wavelet level, thus having 4 sub bands. The approximation image decomposition is divided into four sections and divides into $5 \times 5$ windows for extracting texture features in each section. The advantage of the proposed RBBM is to reduce the $5 \times 5$ window to a $3 \times 3$ window. The proposed RBBM is going to use 17 out of the 25 pixels from the $5 \times 5$ blocks of pixels, by using eight compass directions from the central pixel cp, indexed as shown in Figure 1.

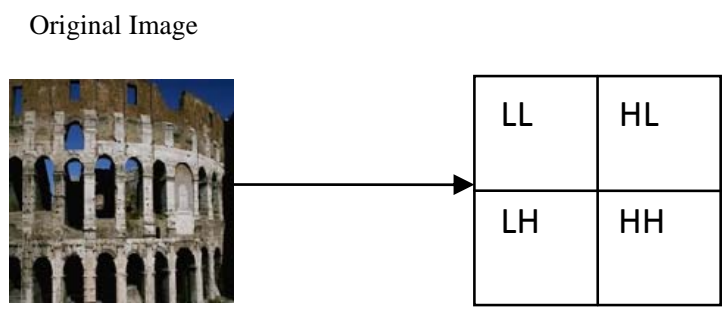

$$
\left(\begin{array}{ccccc}
b 0 & - & b 2 & - & b 4 \\
- & b 1 & b 3 & b 5 & - \\
b 14 & b 15 & c p & b 7 & b 6 \\
- & b 13 & b 11 & b 9 & - \\
b 12 & - & b 10 & - & b 8
\end{array}\right)
$$

Pixel intensity values for $5 \times 5$ window

The average intensity of the pixels along each direction is compared with that of the central pixel in the reduction. The reduction of $5 \times 5$ window to $3 \times 3$ window can be mathematically done by using Equation (1). 


$$
\left\{\begin{array}{l}
2, \text { if } b_{2 i}^{w}+b_{2 i+1}^{w}-2 c_{p}^{w}>0 \\
1, \text { if } b_{2 i}^{w}+b_{2 i+1}^{w}-2 c_{p}^{w}=00 \leq i \leq 7 \\
0, \text { if } b_{2 i}^{w}+b_{2 i+1}^{w}-2 c_{p}^{w}<0
\end{array}\right.
$$

\section{IV.REGION BASED HSV COLOR QUANTIZATION}

The quantization of the number of colors into several bins is done in order to decrease the number of colors used in image retrieval, J.R. Smith [19] designs the scheme to quantize the color space into 166 colors. Li [20] design the non-uniform scheme to quantize into 72 colors. The proposed method used HSV(Hue, Saturation and Value) Color space, because it is natural and is approximately perceptually uniform. the main method of representing color information of images in CBIR systems has been through color histograms. But in our approach, we have represented color in the HSB color space, as floating point values. Each section in an image has six values representing color. These six values represent the average hue, average saturation, average brightness, hue variance, saturation variance and brightness variance respectively, We propose the scheme to produce 15 non-uniform colors. The formula that transfers from RGB to HSV is defined as below:

$$
\begin{aligned}
& H=\left\{\begin{array}{l}
60\left(0+\frac{G-B}{C_{\max }-C_{\min }}\right), \text { if } C_{\max =R} \\
60\left(2+\frac{B-R}{C_{\max }-C_{\min }}\right), \text { if } C_{\max =G} \\
60\left(4+\frac{R-G}{C_{\max }-C_{\min }}\right), \text { if } C_{\max =B}
\end{array}\right. \\
& \mathrm{S}=\frac{C_{\max }-C_{\min }}{C_{\min }} \\
& \mathrm{V}=C_{\max }
\end{aligned}
$$

Where $C_{\max }=\operatorname{Max}(\mathrm{R}, \mathrm{G}, \mathrm{B})$ and

$$
C_{\text {min }}=\operatorname{Min}(\mathrm{R}, \mathrm{G}, \mathrm{B})
$$

The R, G, B represent red, green and blue components respectively with value between $0-255$. In order to obtain the value of $\mathrm{H}$ from $0^{\circ}$ to $360^{\circ}$, the value of $\mathrm{S}$ and $\mathrm{V}$ from 0 to 1 , we do execute the following formula:

$$
\begin{aligned}
& \mathrm{H}=\left(\left(\mathrm{H} / 255^{*} 360\right) \bmod 360\right. \\
& \mathrm{V}=\mathrm{V} / 255 \\
& \mathrm{~S}=\mathrm{S} / 255
\end{aligned}
$$

\section{Proposed Method (RBBM)}

This paper proposes a new technique RBBM for CBIR using boundary based method which we applied in this is wavelet Domain by using 1D Haar Wavelet and for region based we applied HSV color quantization. The block diagram of the proposed method is shown in Figure 2. The proposed method works on the basic principle of the query image which is fed into the convertor for converting of RGB into HSV based on the formula which is presented in the equations 2,3 \& 4.the conversion of RGB to HSV involves dividing of the query image into nine equal sections. For each section we want to calculate the HSV.After the calculation part was completed we want to find the overall averages values of the above sections and store their final resultant. For the same query image which was fed into the region method was now adopted for the boundary based method for finding the haar based wavelet domain which gives threshold value T.Now the same methods are applied to the database image which we want to retrieve the images. The averages values of $\mathrm{H}, \mathrm{S}, \mathrm{V}$ and $\mathrm{T}$ are compared with the database image. If all the values are matched then those images are retrieved and stored.Comparisions will be carried out by the similarity measurement. 


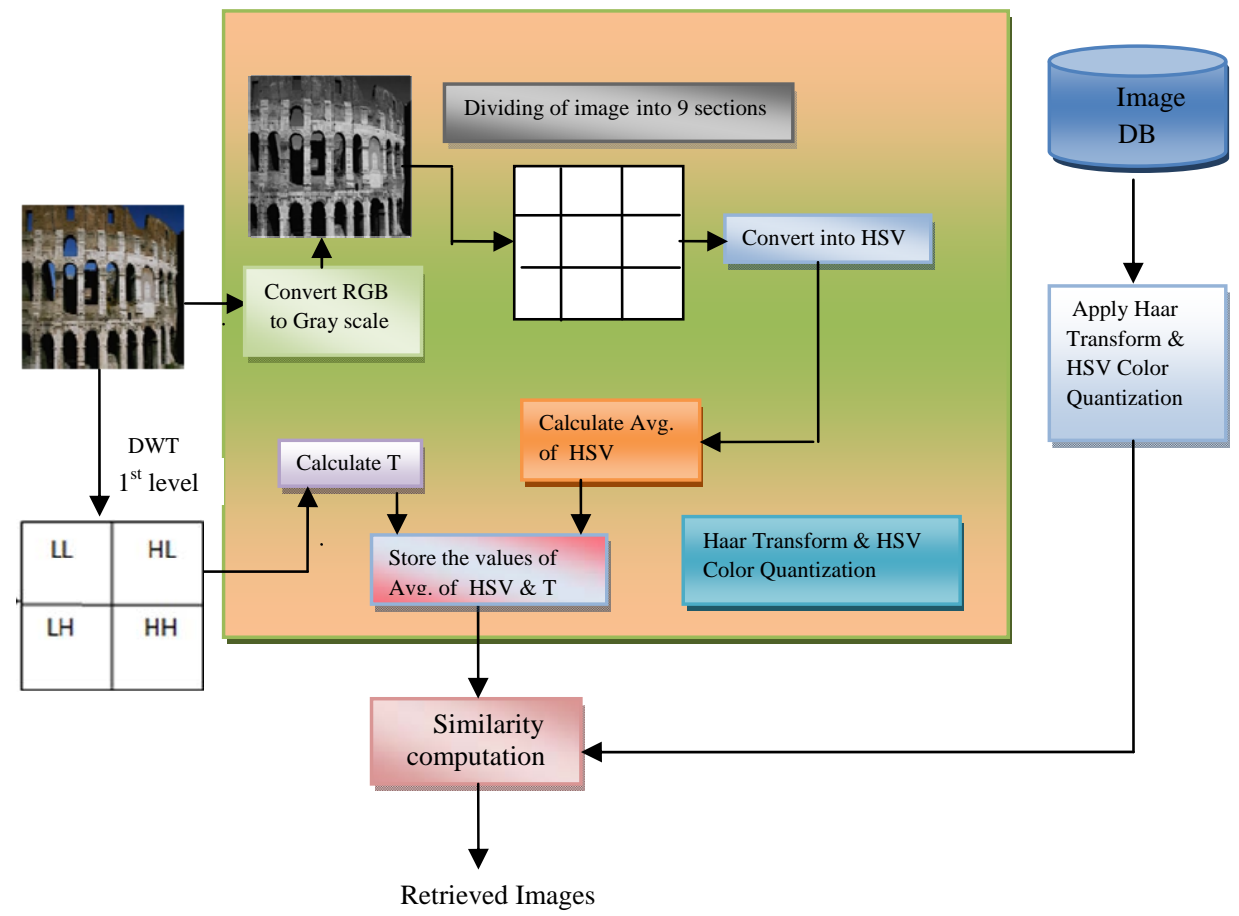

Fig. 2 Block diagram of proposed RBBM

Steps involved in proposed method RBBM

1. Read the query image

2. Convert RGB to Gray

3. Divide image into 9 sections

4. Calculate HSV for each section by using the formula which is mentioned in equation (2), (3) \& (4).

5. Find Average $\mathrm{H}$, Average $\mathrm{S}$, Average V.

6. Apply Haar wavelet transform to the query image.

7. Calculate the average of pixels values and call it as $\mathrm{T}$

8. Compare the Values of H,S, V \& T with the database image.

9. Retrieve the image which is similar to the query image value.

\section{Vi. Performance Evaluation}

To evaluate the retrieval efficiency of the proposed system, we use the performance measure, Recall and precision. Recall measures the ability of the system to retrieve all the models that are relevant, while precision measures the ability of the system to retrieve only the models that are relevant.

Using precision and recall to measure the accuracy of retrieval system is still the most prominent technique.

Precision $=\frac{R_{r}}{T_{r}}$

Recall $=\frac{R_{r}}{T}$

Where

$\mathrm{R}_{\mathrm{r}}$-Represents the number of appropriate images retrieved.

$T_{r}$-Represents the entire images retrieved

T-Represents the total appropriate images retrieved.

Precision and recall results are tabulated in table 1.

\section{CANBERRA DISTANCE}

It is the sum of absolute values of the differences between ranks divided by their sum, thus it is a weighted version of the distance. It examines the sum of series of a fraction difference between the coordinates of a pair. Each term of fraction difference has value lies between 0 \& 1 . The Canberra Distance itself is not between 0 \& 1. If one of the coordinator is 0 , the term become unity regardless the other value, thus the distance not be affected. Note that if both coordinate are 0 's, we need to be defined as $\frac{0}{0}=0$. This distance is very sensitive to a small change when both coordinate near to zero. 


$$
\mathrm{D}_{\mathrm{mn}}=\sum_{k=1}^{i} \frac{\left|x_{m k}-x_{n k}\right|}{\left|x_{m k}\right|+\left|x_{n k}\right|}
$$

For finding the calculation input the values of query image compare it with Database image and store the resultant. From the resultant values we can able to know the matching relevant images. The retrieval result for building is illustrated in Fig.5.The distance metrics used is Canberra distance. When an image is addressed, a searching process will retrieve the closest image $s$ in the database. The retrieved images are displayed according to their distance with the image query. The image that indicating perfect match will be retrieved first. Table 1 depicts the retrieval results for the sample images taken from the Corel dataset. The average precision is 8.11 and the average recall 2.57 .

Table 1: Summarize the Precision and Recall results of the proposed Method RBBM

\begin{tabular}{|l|c|c|}
\hline Category of Images & Average Precision & Average Recall \\
\hline Mountain & 0.853 & 0.321 \\
\hline Dinosaur & 0.871 & 0.187 \\
\hline Building & 0.759 & 0.158 \\
\hline Elephant & 0.901 & 0.254 \\
\hline Flowers & 0.613 & 0.219 \\
\hline Train & 0.941 & 0.298 \\
\hline Castle & 0.815 & 0.18 \\
\hline Cloud & 0.702 & 0.267 \\
\hline Fruits & 0.765 & 0.327 \\
\hline Horses & 0.899 & 0.296 \\
\hline Average (\%) & 0.811 & 0.257 \\
\hline
\end{tabular}

Graph representing the recital scrutiny of texture feature via RBBM

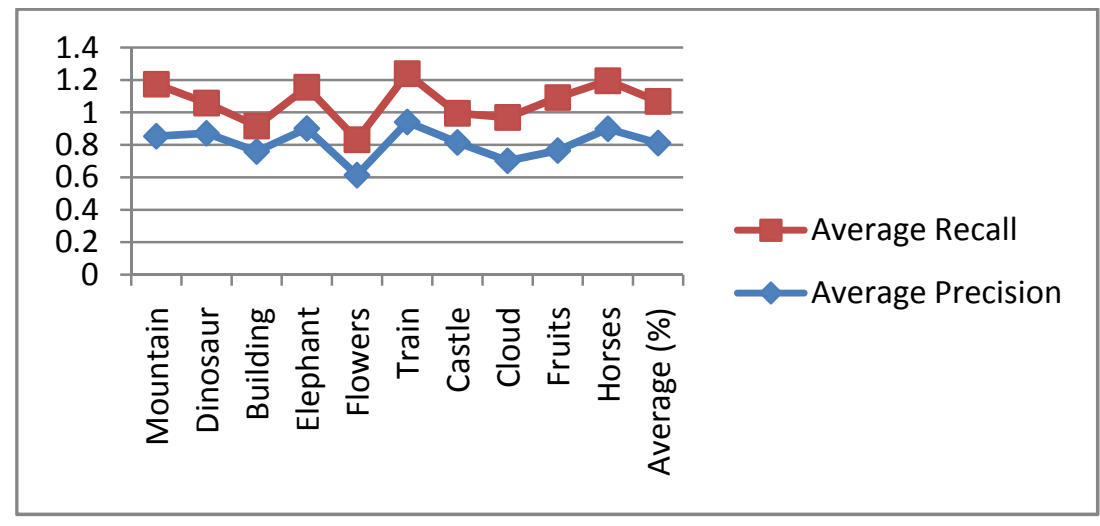

\section{EXPERIMENTAL RESULTS}

We followed an approach to carry out an assessment and modifications to the retrieval of images based on the features experienced by means of a cautiously particular division of the Corel image dataset and the vector space likeness compute. We selected random sample of images from the Corel collection to carry out the distance vector calculation for the retrieval process. We have categorized the images in various forms like Mountain, Building, Dinosaur, Horses, Castle, Cloud, Elephant, Fruits, Flowers, Train etc.We reorganized the Corel sample dataset as many images with similar concepts were not in the same group and some images with different images were in the same group in the original database, each group includes more than 100 images and the image group are category-homogeneous. These concept groups were used in the evaluation of the results of our technique. For each feature we evaluated performance in the configuration described to improve performances were devised and evaluated. The general themes considered were how best to represent an entire image, how to accommodate differing sizes and scale of images and how to cope with the regional qualities of textures. These evaluations were run on the Corel data. The best performing features from the initial evaluation were then tested on the image data set. Tests were run with each texture feature combined with a high performing colour feature. 

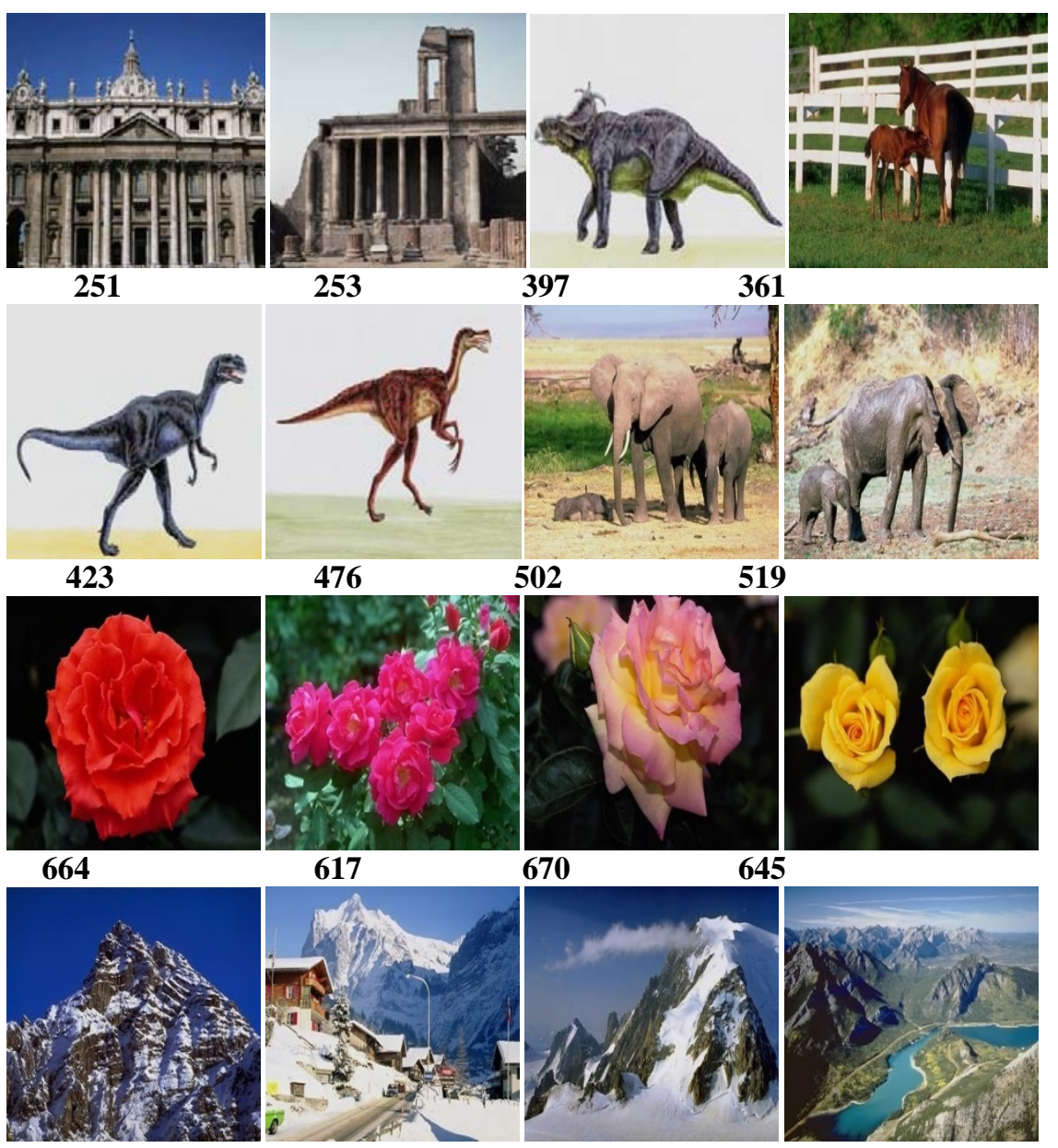

$670 \quad 645$

821 808
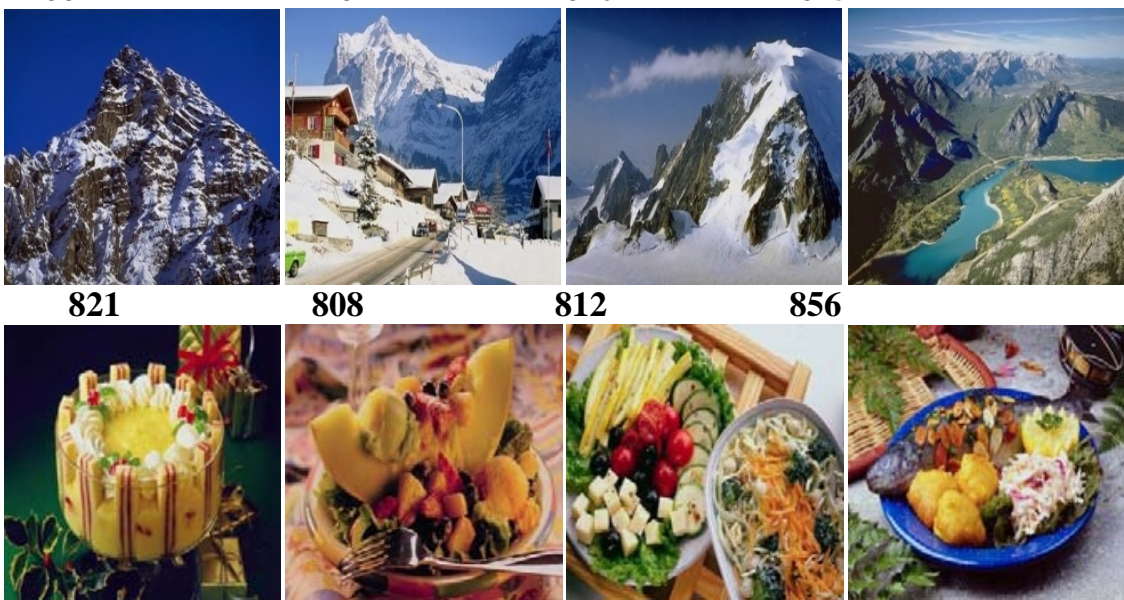

923

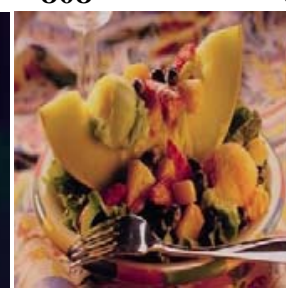

891

812

856

Fig.3 Sample of WANG Image database

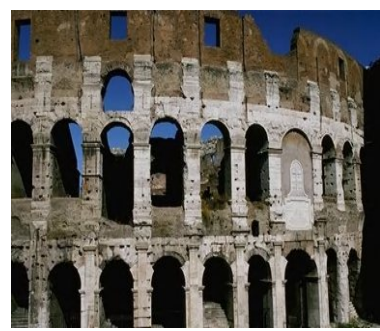

Fig.4 Query Image: Building

Figure 4 shows the query image Building and retrieved images based on the query images from WANG database are shown in Figure 3. Table 1 summarizes the experiment results of proposed RBBM with ten different categories of images. Proposed method retrieved Building images accurately. The overall performance of proposed method obtained more than $81 \%$ of retrieval images with more accurate, efficient and wellorganized. 


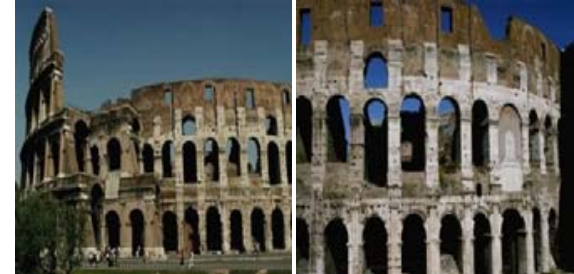

202

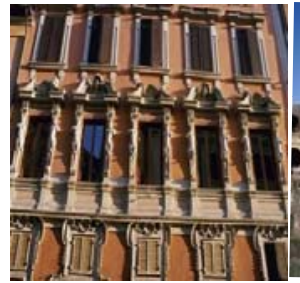

245

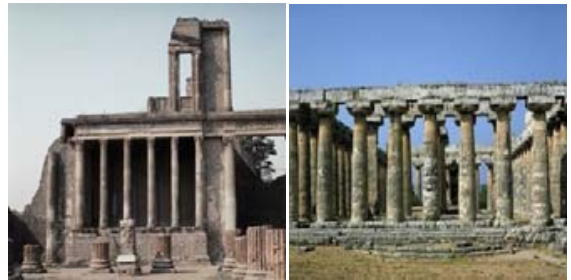

253
212

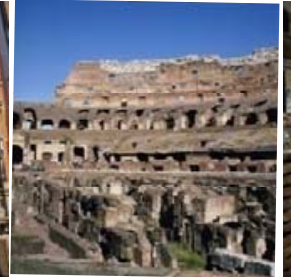

256

267
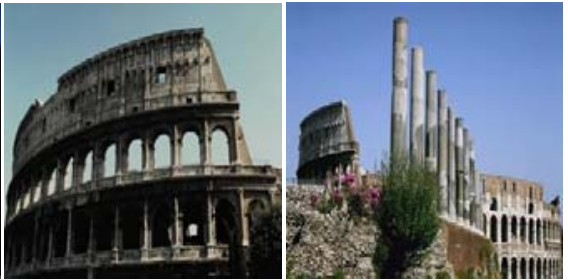

238

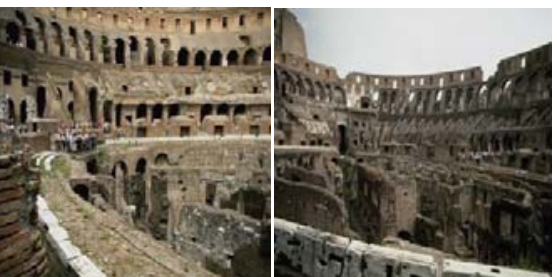

276

231

Fig.5 Retrieved images based on query image Building

The proposed method is examined with the image database named Corel and tested the performance of the proposed method retrieved images. We can take various classes of images present in the image database consisting of various orientations resulting in a total of 1000 images. Figure 3 shows the sample image taken from the image database Corel. The proposed method RBBM is presented in the table showing the average values of precision and recall. Figure 4 shows the query image building and retrieved images based on the query images from the image database are depicted in Figure 5.the precision and recall values are calculated by using the above mentioned formulas in roman IV (performance evaluation).

\section{Assessment with other methods}

The comparison of the proposed methods is carried with J.Sreedhar et.al [10], Manjusha.S et.al [14]. HSV and DWT retrieval methods are compared with the proposed method RBBM. Our proposed method RBBM is compared with other methods which are depicted in the comparison Table2.This proposed method shows there will be some variation in average recall and precision which differs with other methods. Proposed method retrieved Building images accurately. The overall performance of proposed method obtained more than $81 \%$ of retrieval images with more accurate, efficient and well- organized. The experimental results show the accuracy of retrieval when compared to other approaches. Overall, our proposed method RBBM has reduced steps effectively by using wavelet transformation. It shows better performance by the inclusion of region and boundary based methods for a good result in retrieval process of CBIR system. Our proposed RBBM employ these two different techniques collectively to give the accurate retrieval from large database with rapidity. 
Table 2.Comparision table of projected RBBM with further methods

\begin{tabular}{|l|l|l|l|l|l|l|}
\hline \multirow{2}{*}{$\begin{array}{l}\text { Category } \\
\text { of Images }\end{array}$} & \multicolumn{2}{|l|}{ DWT } & \multicolumn{2}{l|}{ HSV } & \multicolumn{2}{l|}{ Proposed RBBM } \\
\cline { 2 - 7 } & Precision & Recall & Precision & Recall & Precision & Recall \\
\hline Mountain & 0.805 & 0.242 & 0.864 & 0.25 & 0.898 & 0.255 \\
\hline Dinosaur & 0.891 & 0.291 & 0.851 & 0.281 & 0.856 & 0.301 \\
\hline Building & 0.799 & 0.257 & 0.801 & 0.245 & 0.841 & 0.269 \\
\hline Elephant & 0.841 & 0.229 & 0.809 & 0.238 & 0.921 & 0.248 \\
\hline Flowers & 0.833 & 0.347 & 0.886 & 0.333 & 0.837 & 0.355 \\
\hline Train & 0.825 & 0.266 & 0.847 & 0.274 & 0.833 & 0.278 \\
\hline Castle & 0.807 & 0.254 & 0.839 & 0.282 & 0.841 & 0.299 \\
\hline Cloud & 0.873 & 0.273 & 0.845 & 0.279 & 0.875 & 0.298 \\
\hline Fruits & 0.868 & 0.241 & 0.851 & 0.252 & 0.874 & 0.247 \\
\hline Horses & 0.809 & 0.204 & 0.805 & 0.216 & 0.839 & 0.248 \\
\hline $\begin{array}{l}\text { Average } \\
\text { (\%) }\end{array}$ & 0.835 & 0.260 & 0.839 & 0.265 & 0.861 & 0.279 \\
\hline
\end{tabular}

Assessment chart with Precision

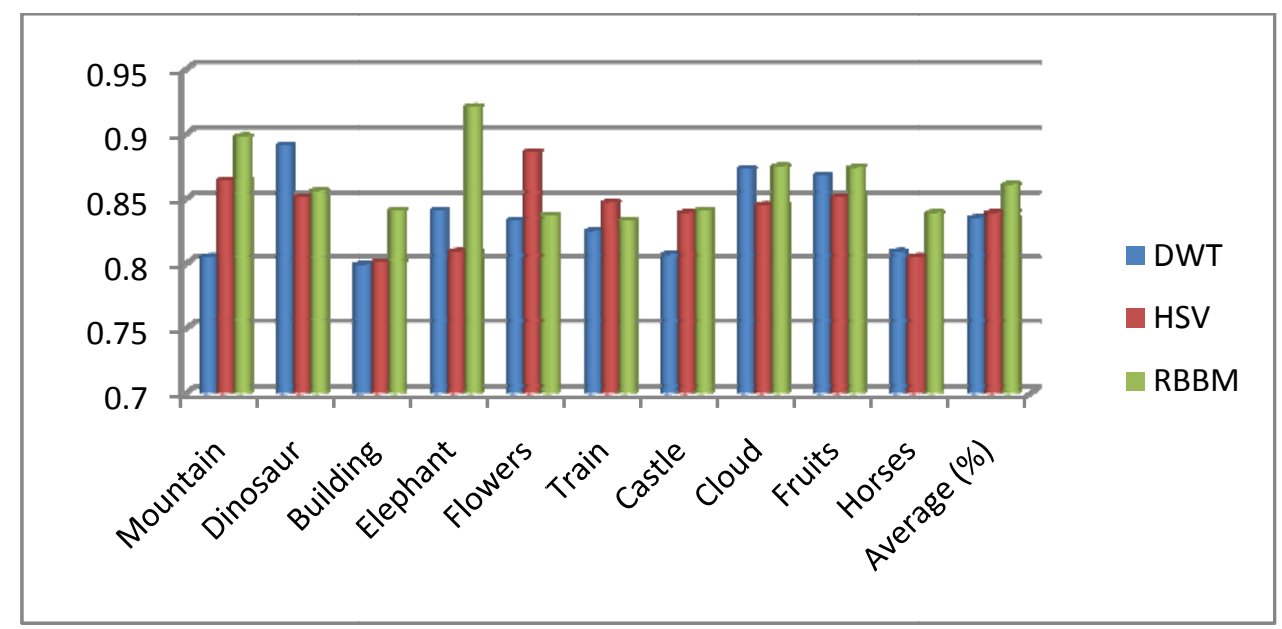

Assessment chart with Recall

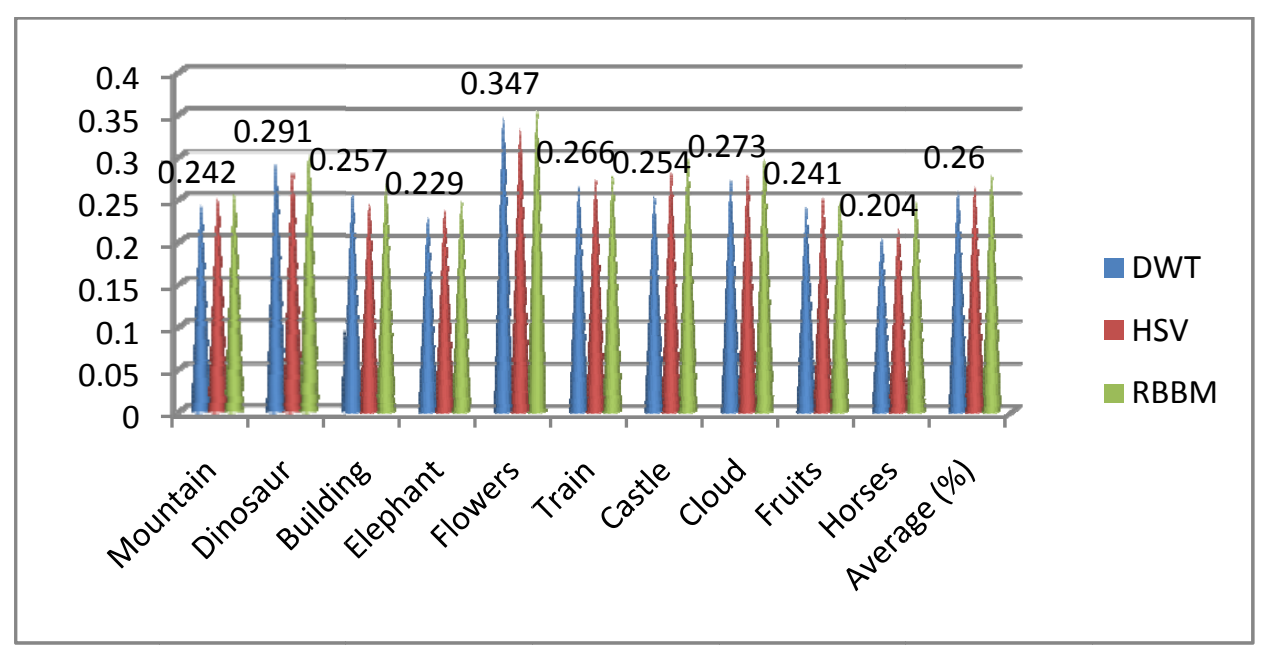




\section{CONCLUSION}

The projected method RBBM has carried out its experimentation with large number of dataset. After applying our proposed method RBBM one can able to find out the value of precision and recall of the images present in the database. The outcome of the precision and recall value compared with other methods. Overall, our proposed method RBBM has reduced steps effectively by using wavelet transformation. It shows better performance by the inclusion of region and boundary based methods for a good result in retrieval process of CBIR system.

\section{ACKNOWLEDGMENT}

The authors would like to express their gratitude to Management of Saveetha University and Chaitanya group of Institutions for providing necessary Infrastructure to carry over the research work.

\section{REFERENCES}

[1] Manimala Singha and K.Hemachandran "Content based image retrieval using color and texture”, in signal \& image processing: An international journal (SPIJ) Vol.3, No.1, February 2012.

[2] Michael S. Lew, Nicu Sebe, Chabane Djeraba, and Ramesh Jain, “Content-based multimedia information retrieval: State of the art and challenges,” ACM Trans. Multimedia Comput. Commun. Appl., vol. 2, no. 1, pp. 1-19, 2006.

[3] R. C. Gonzalez, R. E. Woods and S. L, Eddins. Digital Image Processing Using MALAB, By Pearson Education, 2008.

[4] S. Ardizzoni, I. Bartolini, and M. Patella, "Windsurf: Region based Image Retrieval using Wavelets”, In IWOSS’99, pp. 167-173, 1999.

[5] S. Livens, P. Scheunders, G. V. D. Wouwer and D. V. Dyck, "Wavelets for texture analysis, an overview”, Proceedings of Sixth International Conference on Image Processing and Its Applications, Vol. 2, pp-581-585, 1997.

[6] S.-K. Chang and A. Hsu, Image information systems: Where do we go from here? IEEE Trans. On Knowledge and Data Engineering 4(5 ), 1992.

[7] Dengsheng Zhang, M.I., Aylwin, W. and Lu,G.Content Based Image Retrieval Using Gabor Texture Features”. Proc.1st IEEE Pacific Ri.(2004)

[8] Fischer, S., Cristobal, G., and Redondo, R. 'Sparse over complete Gabor wavelet representation based on local

[9] J.Sreedhar,S.Viswanadha Raju,A.Vinaya Babu,”Query Processing for Content based Image retrieval”, IJSCE, Vol-1_Issue-5.

[10] Sylvain Fischer, Laurent Perrinet, 'Self-Invertible 2D Log-Gabor Wavelets', International Journal of Computer Vision 75(2), 231-246, 2007.

[11] ShamikSural, S. (2005). Histogram generation from the HSV color space using saturation projection. In S. Deb (Ed.), Multimedia systems and content-based image retrieval.Hershey, PA: Idea Group Publishing.

[12] M. Peura and J. Iivarinen, "Efficiency of simple shape descriptors," in Proc. 3rd InternationalWorkshop on Visual Form (IWVF3), May 1997.

[13] ManjushaS , Nelwin Raj N R,”Content Based Image Retrieval Using Wavelet Transform and Feedback Algorithm “,International Journal of Innovative Research in Science, Engineering and Technology, ISSN (Online) : 2319 - 8753.

[14] Huang, J., Kumar, S.R., Mitra, M., Zhu, W.J., Zabih, R.: Spatial color indexing and applications. Int. J. Comput. Vision 35, 245-268 (1999)

[15] Jain, A., Vailaya, A.: Image retrieval using color and shape. Pattern Recognition 29,1233- 1244 (1996)

[16] Saykol, E., Gudukbay, U., Ulusoy, O.: A histogram-based approach for object-based query-by-shape-and-color in multimedia databases. Technical Report BU-CE-0201,Bilkent University, Computer Engineering Dept (2002)

[17] Caputo, B., Dorko, G.: How to combine color and shape information for 3d object recognition: kernels do the trick (2002)

[18] Diplaros, A., Gevers, T., Patras, I.: Combining color and shape information for illumination-viewpoint invariant object recognition 15, 1-11 (2006)

[19] Pala, S.: Image retrieval by shape and texture. PATREC: Pattern Recognition.Pergamon Press 32 (1999).

[20] j.Wang G. Wiederhold,O.Firschein and S.We”Content based Image indexing and searching using Daubechies wavelets”,International Journal on Digital Libraries(IJODL)I,(4)pp 311-328,1998.

\section{AUTHOR PROFILE}

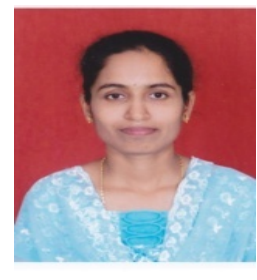

MMrs.R.Tamilkodi received her MCA degree in Bharathidasan University, Trichy, in 2002, and M.Tech (IT) from Karnataka University, Karnataka in 2010.She is working towards her Ph.D at Saveetha University, She is with Godavari Institute of Engineering \& Technology, A.P as an Associate Professor. She has 11 Years of experience in teaching undergraduate students and post graduate students. Her research interests are in the areas of image processing, content based image retrieval and cloud computing. She is a life member in ISCA and Red Cross Society.

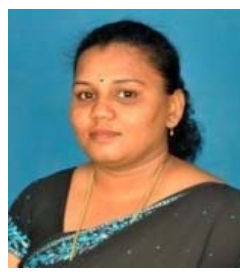

Dr. G Rosline Nesa Kumari received her Ph.D degree in Computer Science and Engineering at Dr MGR University Chennai, and received her M.E. Degree from Sathyabama University Chennai in 2005. She is having Thirteen Years of teaching experience. At present she is working as a Professor, Department of Computer Science and Engineering in Saveetha University, Chennai. She published sixty research publications in various International, National Conferences and Journal. She is a life member of Indian Science Congress Association (ISCA), IAENG, IET, CSI (Life Member), Red Cross. Her research interest includes Image processing, Steganography, Network security and Information Security. 


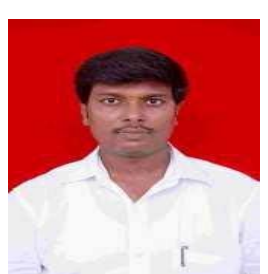

Dr.S.Maruthu Perumal received his B.E, in Computer Science and Engineering from Bharathidasan University and M.E in Computer Science and Engineering from Sathyabama University Chennai. He received Ph.D from Dr. MGR University, Chennai. He is having Fifteen years of teaching experience. At present he is working as a Professor NBKR Institute of Science and Technology, Vidyanagar, Nellore, AP. He published Fifty seven research publications in various Inter National, National Conferences and Journal. His research interest includes Image processing, Digital Watermarking, Steganography and Security. He is a life member of ISCA, IAENG and Institutional Member of CSI. 